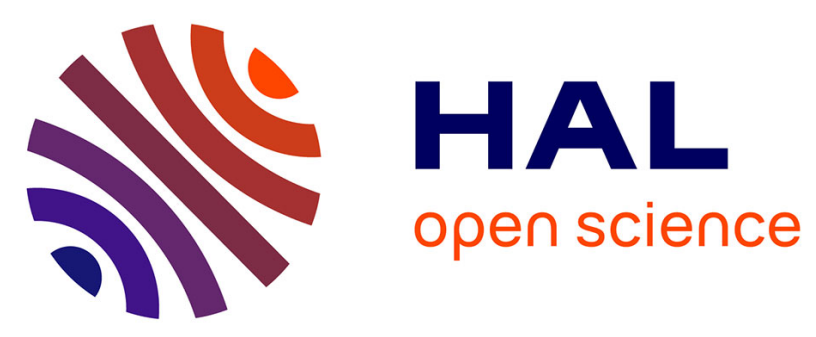

\title{
Construction de l'authenticité et expérience patrimoniale. Conception et réception de la réplique de la grotte Chauvet - Pont d'Arc
}

\author{
Mélanie Duval, Christophe Gauchon, Charlotte Malgat
}

\section{To cite this version:}

Mélanie Duval, Christophe Gauchon, Charlotte Malgat. Construction de l'authenticité et expérience patrimoniale. Conception et réception de la réplique de la grotte Chauvet - Pont d'Arc. Culture et Musées, 2020, Musées et mondes numériques, 35, pp.243-274. 10.4000/culturemusees.5041 . hal02883542

\section{HAL Id: hal-02883542 \\ https://hal.science/hal-02883542}

Submitted on 29 Jun 2020

HAL is a multi-disciplinary open access archive for the deposit and dissemination of scientific research documents, whether they are published or not. The documents may come from teaching and research institutions in France or abroad, or from public or private research centers.
L'archive ouverte pluridisciplinaire HAL, est destinée au dépôt et à la diffusion de documents scientifiques de niveau recherche, publiés ou non, émanant des établissements d'enseignement et de recherche français ou étrangers, des laboratoires publics ou privés. 
Duval Mélanie, Gauchon Christophe, Malgat Charlotte, 2020, Construction de l'authenticité et expérience patrimoniale. Conception et réception de la réplique de la grotte Chauvet-Pont d'Arc, Culture \& Musées, 35, 243-274.

\title{
CONSTRUCTION DE L'AUTHENTICITE ET EXPERIENCE PATRIMONIALE.
}

\section{CONCEPTION ET RECEPTION DE LA REPLIQUE DE LA GROTTE CHAUVET-PONT D'ARC}

\author{
Auteurs : Mélanie Duval, Christophe Gauchon, Charlotte Malgat
}

Mots clefs: Authenticité, fiction, expérience patrimoniale, réplique «Grotte Chauvet 2 Ardèche »

Cet article s'inscrit dans la continuité de plusieurs projets de recherche ayant comme objectif transversal d'analyser les effets de la découverte de la grotte Chauvet-Pont d'Arc dans la reformulation des dynamiques patrimoniales et touristiques du sud-Ardèche. Depuis 2002, ces recherches ont donné lieu à une vingtaine de campagnes de terrain (une à deux campagnes annuelles de deux semaines en moyenne), composées d'observations de terrain, d'entretiens semi-directifs, d'analyse de la littérature grise, de plusieurs enquêtes auprès des visiteurs du sud-Ardèche. Selon des approches complémentaires, deux recherches doctorales ont eu lieu sur ce terrain (Duval, 2007 ; Malgat, 2016), ainsi que plusieurs publications (entre autres : Cachat et al., 2012 ; Duval, 2011 ; Duval \& Gauchon, 2013 ; Duval et al., 2019 ; Malgat \& Duval, 2014 ; Malgat et al., 2015, 2012 ; Gauchon, 2009). Les résultats exposés dans le présent article s'appuient sur des données obtenues au sein du programme de recherche «Singulariser les Territoires de Montagne », 2015-2018, financement Labex Item, lequel programme a permis la réalisation d'une enquête auprès des visiteurs de la réplique de la grotte Chauvet-Pont- d'Arc (deux campagnes, respectivement en août 2016 et en août 2017), et la conduite de nouveaux entretiens semi-directifs avec les acteurs territoriaux (2015, 2016 et 2017).

Ce travail a notamment été réalisé grâce au soutien financier du LABEX ITEM (ANR-10LABX-50-01) dans le cadre du programme "Investissements d'Avenir" géré par l'Agence Nationale de la Recherche, projet de recherche "Singulariser les Territoires de Montagne » porté par Karine Basset et Véronique Peyrache-Gadeau.

\section{Résumé :}

Dans le champ des patrimoines culturels, le recours à des copies, partielles ou intégrales, donne aux visiteurs la possibilité de voir et d'accéder à des objets ou des lieux difficiles d'accès, voire fermés. Dans le cas des grottes ornées, où les copies sont réalisées dans un souci de perfection, avec la volonté de restituer des images et de transmettre les émotions que les visiteurs auraient devant les peintures originales, les questions de l'authenticité et de l'expérience patrimoniale se posent. Quels sont les procédés mobilisés par les concepteurs en vue de faire éprouver l'authenticité ? Dans quelle mesure le dispositif scénographique déployé conduit-il les visiteurs à faire une expérience authentique de ce qui fait patrimoine ? Cette problématique de la construction de l'authenticité et de l'expérience patrimoniale sera travaillée à partir de l'analyse de la réplique de la grotte Chauvet-Pont d'Arc, située à proximité de l'originale dans le sudArdèche (France). Après avoir discuté la place de l'authenticité et de l'expérience dans ce qui fait patrimoine, l'article analyse les modalités de construction puis de réception de la fiction élaborée, en observant dans quelle mesure les visiteurs se laissent prendre au jeu du demi-croire 
(Lambert, 2013) et de l'enchantement proposé par les concepteurs (Winkin, 1998, 2001). In fine, ces réflexions dépassent la seule thématique des grottes ornées et amènent à reformuler la place de l'expérientiel dans la construction d'un rapport patrimonial (Tornatore, 2019).

\section{Introduction}

La conciliation des enjeux de préservation et de mise en tourisme des sites culturels bénéficie depuis les années 1990 du développement des technologies digitales et des avancées en matière de production de modèles 3D (Pieraccini et al., 2001 ; Stylianidis \& Remondino, 2016). Pour le seul champ des sites d'art rupestre, un gradient s'observe et les combinaisons sont multiples (Pinçon \& Geneste, 2010), allant jusqu'à recréer la sensation de l'original avec la réalisation de copie «parfaite » s'appuyant sur des relevés 3D, comme c'est le cas pour la grotte Chauvet Pont d'Arc (Delannoy et al., 2014), mais également pour Lascaux ou Altamira (Kaminski, 2014 ; Leresche, 2020). Dans ce cas de figure, où les copies sont réalisées dans un souci de perfection, avec la volonté de restituer des images et de transmettre les émotions que les visiteurs auraient devant les peintures originales, la question de l'expérience patrimoniale se pose (Duval et al., 2019). Quels sont les procédés mobilisés par les concepteurs en vue de faire éprouver l'authenticité ? Dans quelle mesure les caractéristiques de la réplique et le dispositif scénographique déployé conduisent-ils les visiteurs à faire une expérience authentique de ce qui fait patrimoine?

Recoupant la dialectique du vrai et du faux $(E c o, 1985)$ et la problématique de l'hyper-réalité (Baudrillard, 1981), ces questionnements appellent une réflexion de fond sur la place de l'authenticité et celle du volet expérientiel dans la construction d'un rapport patrimonial (Tornatore, 2019). Les processus d'enchantement (Lallement \& Winkin, 2015; Winkin, 2001,1998) de suspension volontaire de la crédulité et de déni du langage (Lambert, 2013) offrent des clefs de lecture pertinentes pour analyser les dynamiques à l'œuvre. Ces éléments conceptuels posés, notre propos se centrera sur la copie de la grotte Chauvet-Pont d'Arc. Objet hybride croisant des impératifs de développement économique et de médiation culturelle, « La Caverne du Pont d'Arc », renommée la « Grotte Chauvet 2 -Ardèche » en février $2019^{1}$, ouvre en avril 2015, à 2 km à vol d'oiseau de la grotte Chauvet-Pont d'Arc (Cachat et al., 2012). Le site se compose de plusieurs bâtiments auxquels un billet d'entrée unique permet d'accéder ${ }^{2}$. En quatre ans d'exercice, 2 millions de visiteurs se sont rendus sur le site, 420000 pour l'année $2018^{3}$.

Dans la continuité d'un précédent article interrogeant en quoi la matérialité de la copie permettait un transfert de patrimonialité (Malgat et al., 2015), l'objectif est ici d'analyser la rencontre entre des dispositifs scénographiques visant à construire de l'authenticité et les modalités de réception par les visiteurs, et d'interroger dans quelle mesure ces derniers font une expérience de ce qui fait patrimoine (à la différence de «ce qui est patrimoine » (Davallon, 2006 ; Flon, 2012). In fine, ces réflexions dépassent la seule thématique des grottes ornées et amènent à questionner plus largement la place du ressenti de l'authenticité dans la construction d'un rapport patrimonial, dans une ontologie sociale des patrimoines (Leveau, 2018).

\section{Positionnement conceptuel : authenticité, enchantement, expérience patrimoniale}

La patrimonialisation repose sur l'attribution et le partage de valeurs patrimoniales, lesquelles sont fonction de critères qui évoluent dans le temps et selon les contextes socio-culturels (Smith, 2006). Parmi ces derniers, l'examen des règlements, des procédures ou encore des pratiques des institutions patrimoniales souligne le caractère central de l'authenticité dans la définition de ce qui fait patrimoine (Labadi, 2010). L'authenticité fait figure de condition sine qua non dans l'entreprise institutionnelle de définition de ce pourquoi tel bien fait patrimoine, comme l'atteste, à titre d'exemple, la procédure d'inscription d'un bien sur la liste du patrimoine 
mondial qui commence, invariablement par la «déclaration d'authenticité » du bien candidat, indépendamment des autres valeurs attribuées à ce dernier. A charge pour les candidats à l'inscription au Patrimoine mondial de démontrer l'authenticité des biens candidats, qu'ils sont bien ce qu'on pense qu'ils sont. Cette manière d'appréhender l'authenticité relève d'une ontologie substantialiste du patrimoine, où l'authenticité est liée à la réalité matérielle de l'objet. Dépassant cette entrée, Leveau propose d'aborder le fait patrimonial dans le cadre d'une ontologie sociale ${ }^{4}$, dans laquelle le patrimoine se définit comme un objet mouvant, production de réseaux sociaux qui concourent à sa qualification (Leveau, $2018: 60$ ). Cette approche amène à aborder l'authenticité comme un construit social hybride (Jones, 2009), couplant les caractéristiques matérielles et immatérielles de l'objet, qu'elles soient réelles ou fictionnelles, et l'ensemble des perceptions et émotions à travers lesquelles l'élément en question recouvre une puissance symbolique. L'authenticité s'appuie à la fois sur l'objet lui-même, la certification de sa provenance, le lien indiciel établit avec les contextes culturels et historiques dont il provient, ainsi que sur un ensemble de dynamiques sociales et communicationnelles. Cela se vérifie pour des monuments restaurés, telles que des cathédrales (Jones, 2009) ; c'est également le cas pour des objets archéologiques exposés dans des vitrines de musée ou pour des œuvres d'art. Ces derniers ont bien souvent fait l'objet de traitements chimiques complexes afin de pouvoir être exposés aux publics sans subir les morsures du temps. La perception de leur authenticité relève à la fois des propriétés matérielles intrinsèques de l'objet exposé et d'un processus de négociation entre les individus et le dispositif scénographique mis en place : «si dans son apparence et sa texture, l'objet est semblable à l'état d'origine, sa substance n'en a pas moins été modifiée, parfois radicalement, par les interventions des restaurateurs, qui sait que sa mission consiste à restituer une "image fidèle" de l'objet primitif. A certains égards, toute pièce traitée dans un laboratoire devient donc un "faux" » (Kaeser, 2011 : 22) qui, par un effet de réel (Barthes, 1968) n'en est pas moins perçu comme étant authentique.

De telles pratiques soulignent l'importance du volet expérientiel et du ressenti d'authenticité dans la définition de ce qui est (de ce qui fait !) authentique et pose la question des modalités de construction et de réception de l'authenticité, autrement dit, des dispositifs déployés et de l'adhésion des visiteurs à ces derniers. Le cadre théorique de l'enchantement, développé par Y. Winkin (1998, 2001), couplé aux réflexions sur le demi-croire de F. Lambert (2013) offrent des clefs de lecture pertinentes pour appréhender l'authenticité comme un processus social coconstruit, associant d'une part les «ingénieurs de l'enchantement» (Winkin, 2001 : 172), et d'autre part « des spectateurs pleins de bonne volonté, c'est-à-dire des acteurs qui adhèrent sans barguiner aux règles tacites de la situation» (Lallement \& Winkin, 2015: 118) dans un processus de « suspension volontaire de l'incrédulité » (Coleridge cité par Lallement \& Winkin, 2015 : 118) avec «le bonheur de "sombrer" dans l'authenticité de ce que l'on me raconte » (Lambert, 2013 : 81). Ici, l'authenticité se dissocie de la nature ontologique des objets : que l'objet soit réel, qu'il relève de la fiction, ou présente plus couramment une forme d'hybridité (Schaeffer, 1999), le ressenti d'authenticité résulte d'une rencontre entre des concepteurs et des consommateurs, conjointement impliqués dans des opérations de dénégation et de suspension (Lallement \& Winkin, 2015). Dans une réflexion sur les copies de grottes ornées, il s'agit dès lors d'analyser dans quelle mesure et à quelles conditions la visite d'une réplique permet un ressenti d'authenticité et peut conduire les visiteurs à faire une expérience patrimoniale de l'art pariétal paléolithique.

\section{Dispositifs scénographiques et construction de l'authenticité dans la copie de la grotte Chauvet-Pont-d'Arc La matérialité de la copie}


Dans le champ des monuments culturels, de récentes recherches sur la notion d'authenticité mettent en avant l'importance de la matérialité des copies dans la manière dont les individus font l'expérience et négocient l'authenticité (Jones 2009, 2010 ; Jones \& Yarrow, 2016). En particulier, les effets de patine, exprimant le passage du temps, participent à la création d'un sentiment de pastness (Holtorf, 2013). Ce ressenti participe à la construction d'un rapport au temps, et s'inscrit au cœur des réflexions des archéologues et des scénographes investis dans la re-constitution de lieux archéologiques où l'ambiance, créée à partir de dispositifs matériels, vise à faire éprouver aux visiteurs l'épaisseur du temps (Flon, 2012 ; Stone \& Planel, 1999). Suivant cette logique, les acteurs porteurs du projet de la réplique de la grotte Chauvet-Pont d'Arc ont fait le choix de la matérialité avec la construction de bâtiments : "L'Espace de Restitution de la Grotte Chauvet (ERGC) va permettre au public de voir l'invisible, de découvrir ce patrimoine universel exceptionnel. En tant qu'élément phare, ERGC devient le bien, ce qui va pouvoir se voir. Quand je suis arrivé, il y avait un débat sur : est-ce que c'est bien de faire un équipement de 40 millions d'euros ?, Est-ce qu'on ne ferait pas mieux de valoriser le patrimoine de la grotte Chauvet avec des expositions, des conférences, des spectacles, du virtuel ? Peutêtre, et l'un n'empêche pas l'autre d'ailleurs, mais je reste persuadé que seul un monument central, unique permet de rendre compte des richesses de la grotte » (entretien avec Richard Buffat, directeur du SMERGC, le 12/02/2013).

Cependant, reproduire la grotte Chauvet dans son intégralité $\left(8000 \mathrm{~m}^{2}\right)$ aurait nécessité de trouver une surface foncière disponible importante dans un environnement contraint (pression touristique, périmètres de protection), sans parler des coûts engendrés par un fac-similé stricto sensu. Aussi, c'est finalement une contraction de la grotte originale que le visiteur découvre avec un cheminement dans un espace clos selon 10 stations donnant à voir les fondamentaux de l'original, lesquels sont reproduits à l'identique (Malgat et al., 2015). Empruntant à la fois au registre de l'anamorphose et à celui du fac-similé, la copie de la grotte Chauvet permet aux acteurs territoriaux d'annoncer « une reconstitution parfaite de La Grotte Chauvet Pont-d'Arc » (SMERGC, 2012 : 5) et ce d'autant plus que les choix effectués par le SMERGC dans la phase de sélection des parties à reproduire ont été validés par un comité scientifique, composé, en partie, de scientifiques également impliqués dans l'équipe en charge de l'étude de la grotte.

\section{Un dispositif scénographique immersif}

Les volets expérientiel et émotionnel sont au cœur de la restitution. En écho aux actuelles tendances en muséologie visant le développement d'expériences multi sensorielles (Levent \& Pascual-Leone, 2014), la mise en condition du visiteur par toute une série d'artifices sensoriels vise à fabriquer un ressenti d'authenticité. Les porteurs et réalisateurs du projet, « les ingénieurs de l'enchantement » pour reprendre la terminologie de Y. Winkin (2001), font ainsi le pari que l'émotion suscitée permettra d'entrer en communication avec les ancêtres : «au-delà de la restitution scientifique, l'intérêt du projet était de transmettre l'esprit de la grotte, les émotions ressenties par les chercheurs au moment de la découverte. En deux ans, je suis allée une quarantaine de fois dans la grotte. J'observais beaucoup, j'essayais de repérer les caractéristiques principales de chaque zone pour essayer de restituer cet esprit-là » (interview de Mélanie Claude, scénographe en charge du projet de la réplique, 04/05/2015, France TV Info $^{5}$ ).

L'enjeu est de charger la copie d'une puissance symbolique similaire à celle de l'originale de telle sorte que les visiteurs ressentent la même émotion que s'ils visitaient la vraie... ce qui est d'autant plus aisé à produire que la grotte originale est fermée, inaccessible et que les visiteurs, même s'ils ont pu visiter d'autres grottes ornées, ne sont pas en mesure de comparer l'original et la copie. On est ici dans le registre de la croyance et de la vraisemblance (Lambert, 2013) et l'objectif est de penser un dispositif à même de susciter des émotions suffisamment fortes pour qu'elles puissent être collectivement partagées, de placer les individus dans une logique d'enchantement où «ils ont très envie de se laisser prendre et de partager cette situation de 
capture avec leurs enfants ou leur amis présents, sinon les autres membres du groupe, qui leur sont à peu près inconnus, mais dont ils se sentent solidaires » (Lallement \& Winkin, 2015 : 118).

Dans cette perspective, un soin particulier a été apporté à la reconstruction de l'environnement endo-karstique (parois, sol, plafond, concrétions). Des procédés innovants ont été développés par les plasticiens et les géomorphologues du comité scientifique pour copier le paysage karstique de la grotte Chauvet. Nouvelles techniques de moulages, résines, colorants, effets de suintement des parois, brillance des sols, transparence des draperies (Delannoy et al., 2014 ; Geneste et al., 2017), sont autant d'éléments qui visent à construire une forme d'authenticité, avec comme ambition de créer une grotte ${ }^{6}$ et de donner à ressentir l'épaisseur du temps (Holtorf, 2013) en donnant à voir des peintures datées de 36000 ans BP (Valladas et al., 2001).

Le décor étant posé, le registre sensoriel a été travaillé de manière à créer une atmosphère souterraine: "Pour susciter l'émotion provoquée par la plongée au cœur des temps immémoriaux, les cinq sens sont stimulés. Fraîcheur, humidité, silence, obscurité, sensations olfactives contribueront à immerger les visiteurs dans cet univers si particulier, qui semble encore habité par la présence des hommes et celle des ours » (SMERGC, 2012:9). L'enjeu est ici de garantir une « expérience touristique authentique », de manière à « donner à voir, à vivre l'original » (entretien Pascal Terrasse, député, président du SMERGC, le 22/03/2013), ce qui n'empêche pas des ajustements entre l'environnement climatique de la vraie grotte et celui créé pour les visiteurs dans la copie. Ainsi, le taux d'humidité, initialement de $75 \%$, a été revu à la baisse pour le confort de la visite. Il en va de même pour la température, laquelle varie de 6 et à $8^{\circ} \mathrm{c}$ par rapport à la température extérieure pour donner " un effet de frais ", sans pour autant être à 13 degrés comme c'est le cas à l'intérieur de la grotte Chauvet. Ici, c'est avant tout la vraisemblance et l'effet de réel qui sont recherchés (Barthes, 1968).

L'ambiance feutrée du monde souterrain est travaillée au moyen de l'éclairage ${ }^{7}$ et des casques audio distribués à l'entrée ${ }^{8}$. Ce dispositif permet : 1 / au guide de livrer ses commentaires à voix basse et de canaliser plus facilement son groupe (meilleures conditions de travail), 2/ aux visiteurs de mieux entendre les commentaires tout en les isolant d'éventuels bruits parasites (qualité de la visite), et ce faisant, 3/ de « rentrer dans l'intimité de la grotte » (entretien avec Valérie Molès, Responsable médiation culturelle, pédagogique et scientifique de la réplique, 6 mai 2016) en favorisant «a semi-spiritual relationship with the objet of the gaze» (James, 2016 : 523 ; dimension expérientielle), et enfin 4/ au gestionnaire de gérer très précisément le temps de la visite et le nombre de groupes par heure, étant donné que ce dispositif limite les interactions entre les visiteurs et le guide, lesquels doivent attendre d'être sortis de la copie pour poser leurs questions (rendement économique).

L'impression de visiter une vraie grotte est renforcée par le choix du site (plateau karstique du Razal, et non pas la vallée du Rhône comme ce fut un temps envisagé, Cachat et al., 2012) et la disposition semi-enterrée du bâtiment. Si la copie se trouve dans un bâtiment d'allure très contemporaine, c'est au terme d'une rampe d'accès descendante, traduisant une sensation d'enfoncement dans un espace souterrain et engageant le corps sur un plan kinesthésique, que le visiteur se retrouve dans un premier sas d'entrée de la copie. Là, selon l'heure indiquée sur le ticket d'entrée, les groupes se dirigent vers un second sas où ils sont équipés de casques audio. Le cheminement de la visite les amène alors à un troisième sas où, dans une semipénombre, ils écoutent les premiers commentaires avant que la porte de la reconstitution s'ouvre de manière théâtrale (porte automatique coulissante qui s'ouvre à la manière d'un rideau de théâtre), suscitant des réactions d'émerveillement. «Wahou, c'est beau » accompagne immanquablement l'ouverture de la porte de la part d'un ou plusieurs des visiteurs du groupe. A l'intérieur, la déambulation se fait par des passerelles et des plateformes à distance des reproductions et s'accompagne d'un processus de sacralisation induit par les consignes 
énoncées lors de la distribution des casques audio : ne pas prendre de photos, même sans flash, ne pas faire de films, ne pas toucher les concrétions ni les peintures. Aux dires des gestionnaires du site, "l'interdiction de faire des photos et des films vise avant tout à préserver l'ambiance "grotte". A priori, les flashs n'ont pas de conséquences sur la préservation des moulages » (entretien avec Valérie Molès, 06/05/2016). Dans les faits, cette nuance n'est pas portée à connaissance des visiteurs, des informations contradictoires leur sont même apportées: «Dernière chose avant de commencer mes explications, dedans, on ne touche pas aux parois, et alors là, je suis extrêmement sérieuse, on ne touche pas des œuvres d'art ; les adultes, c'est comme pour les enfants, on touche avec les yeux » (visite du 2 août 2016, 14h25). Les visiteurs sont conditionnés pour aborder la visite de la copie comme si cette dernière était une grotte ornée originale, et nous verrons par la suite dans quelle mesure ils adhèrent à ce double processus du comme si et du je sais bien mais quand même (Lambert, 2013).

\section{Place et rôle des guides officiant dans la copie}

Dans la catégorie des « ingénieurs de l'enchantement» (Winkin, 1998), les guides occupent une place centrale dans la mesure où leur guidage vise directement, et au plus près des visiteurs, à construire un ressenti d'authenticité. Durant les étés 2016 et 2017, 22 visites guidées ont été suivies, enregistrées et anonymées (14 en français, 8 en anglais $)^{9}$. Si leur analyse fait état de variations, notamment dans la manière d'aborder l'explication de certains panneaux, elle met en avant de fortes similitudes dans la manière de gérer les rapports entre fictionnel et réel. Abordée dans le dernier sas précédant l'entrée dans la copie, la problématique de la copie est ensuite reléguée au second plan, si ce n'est occultée. Les guides ${ }^{10}$ décrivent les panneaux et l'environnement karstique comme si c'étaient des originaux, de manière à faire éprouver aux visiteurs le sentiment d'être dans une vraie grotte ornée de peintures datées de 36000 ans BP. Très clairement, l'objectif est de « faire en sorte que la magie puisse opérer (...) Au tout début de la visite, avant de rentrer dans la copie, je conseille aux guides de dire aux visiteurs qu'ils vont rentrer dans une copie, afin de ne pas leur mentir, mais après, je leur conseille aussi de ne plus aborder cette question afin que le charme opère » (entretien avec Valérie Molès, le 06/05/2016).

Lors de la visite, les frontières entre réel et fictionnel sont volontairement brouillées de manière à installer les visiteurs dans la vraie grotte. Les effets du réel sont recherchés : la conjugaison des verbes et le jeu des pronoms (nous) entretiennent l'illusion que les visiteurs sont en face des œuvres produites par les Aurignaciens. Ainsi, les discours tenus à la station 6, devant le panneau au hibou gravé : « Ici, il y a plein d'argile molle, un peu comme du beurre mou, si vous faites l'empreinte de votre doigt, cela reste. Et on va avoir des tracés digités. Et ils vont nous faire une petite merveille, et quelle merveille, la seule et unique représentation au monde d'un hibou grand-duc. Il n'y a aucune hésitation dans ses gestes, il ne reprend jamais ses traits, il lui a fallu environ 5 secondes pour dessiner ce hibou en quelques traits. Regardez la beauté et la sureté du geste, et la fraicheur du trait ! On a encore les empreintes des doigts ; on dirait que cela a été fait il y a 5 minutes alors que cela date d'au moins 21000 ans, avant l'effondrement du porche » (visite du 4 août, 15h30).

L'expérience de l'authenticité semble être de mise, comme l'attestent des remarques enregistrées à la fin des visites. Le 8 août 2016, à la fin de la visite de $10 \mathrm{~h} 25$, un touriste demande si la reconstitution a été faite avec les mêmes techniques que les peintures réalisées par les Aurignaciens. Le guide répond : «Oui, au niveau des dessins, c'est bien de l'ocre et du charbon de bois que vous avez pu voir, réalisés par des artistes contemporains. Par contre, ce ne sont pas des murs de calcaire. Vous, ce que vous avez vu en termes de murs, c'est un grillage 
qui donne les volumes du mur et par-dessus on a projeté du mortier paysager que l'on a peint, gravé, sculpté... » Et de se faire couper la parole par une autre participante du groupe : «non, ne nous dites pas ça, arrêtez, vous cassez la magie! ». Certains visiteurs vont même jusqu'à douter de l'existence de la réplique « mais en fait, là, c'est la vraie grotte et vous l'avez habillée dans un monument moderne? », comme si l'environnement karstique contenant la grotte originale avait été raboté, et qu'un bâtiment contemporain avait été construit par-dessus (Visite du 12 août 2017).

On retrouve ici les opérations de dénégation et d'adhésion volontaire et collective à l'illusion, décrite par Y. Winkin dans son approche anthropologique de l'enchantement : "il ne faut pas rompre le charme, ce dernier mot étant entendu dans son sens fort d'ensorcellement. Les visiteurs sont prêts à lutter, en convoquant aussi souvent que nécessaire la formule "je sais bien mais quand même", contre tout retour à la réalité qui serait provoqué par l'apparition de représentants de l'autre monde » (Lallement \& Winkin, 2015 : 119).

\section{Modalités de réception de la réplique de la grotte Chauvet-Pont d'Arc Les mécanismes du ressenti d'authenticité}

En vue d'interroger la dimension performative du dispositif déployé dans la copie, une enquête auprès des visiteurs a été conduite durant deux campagnes de terrain, août 2016 et août $2017^{11}$. En sus de questions ciblées sur les pratiques touristiques, une partie de l'enquête visait à analyser dans quelle mesure les visiteurs ont un ressenti d'authenticité (Latour \& Lowe, 2011), quels sont les éléments qui, de leur point de vue, concourent à ce ressenti ou au contraire, le limite (Jones 2010) et dans quelle mesure ce ressenti d'authenticité installe les visiteurs dans un rapport patrimonial par rapport au site visité.

La totalité des personnes interrogées sont très satisfaites de la visite effectuée, avec des gradients allant de «J'ai passé un bon moment » (FR_17) à «C'était vraiment superbe, superbe, on en sort enchanté. On est vraiment.... C'était magnifique quoi. Même si on est dans une reproduction, on a une immersion qui est totale, on s'y croit complètement » (FR_38). «Superbe », «magnifique », «enchanté », autant de vocables qui témoignent d'un processus d'enchantement auquel, sans être dupes, les visiteurs se laissent prendre par une «suspension volontaire de l'incrédulité » (Coleridge cité par Y. Winkin, 1998).

La majorité ${ }^{12}$ dit avoir eu le sentiment, à un moment ou un autre, d'être dans une vraie grotte, témoignant de la capacité du visiteur à lâcher prise et faire sien les codes fictionnels qui lui sont proposés. Pour quelques-uns ${ }^{13}$, cette sensation est immédiate : «A partir du moment où les portes s'ouvrent, on est plongé dans la grotte. Rien qu'en hauteur, quand tu vois le plafond, les concrétions, tu ressens la fraicheur, l'humidité, t'es dans la pénombre. Les stalagmites on dirait vraiment des vraies. Ouais t'as direct l'impression d'être dans la vraie grotte » (FR_25).

Pour la majorité d'entre eux, elle intervient au bout de cinq à dix minutes : "C'est même stupéfiant la qualité du travail pour reproduire. Oui on a vraiment l'impression que c'est une vraie grotte, il y a la température, la fraîcheur... il y a l'ambiance. C'est vraiment passionnant, c'est stupéfiant. Au début on se demande comment ils ont fait et puis au bout de 5 mins, on se laisse emporter. Et j' ai même oublié de demander à la fin comment ils avaient fait la réplique » (FR_10); ou encore ce visiteur anglophone «After 10 minutes, we really have the impression to be in dumping in the real cave, we have anymore the sensation than it is a reconstruction, he is completely forgotten » (EN_8).

Enfin, pour $20 \%$ des personnes interrogées, cette impression est partielle : «L'abstraction (sic) de ne pas être dans l'original, cela ne marche pas tout le temps. Quand on est posé devant le panneau et qu'on a le temps de regarder les œuvres dans le détail, oui, on se sent dans la vraie 
grotte. Mais quand un autre groupe arrive, que vous voyez le monde, cela ne fonctionne plus parce qu'on sait qu'on ne peut pas être autant lors d'une visite de vraie grotte »(FR_21). Ici, la situation d'enchantement est mise à mal par les objectifs de rentabilité économique qui imposent des fréquences élevées de rotation des groupes (cf. infra).

Du point de vue des visiteurs, cinq principaux éléments scénographiques concourent au ressenti d'authenticité :

1/ le décor: «Les draperies, les concrétions, elles sont tellement bien faites que tu oublies que tu es dans une copie » (FR_4);

2/ l'ambiance : «On est plongé dans le milieu naturel, on a toutes les sensations, la température, l'obscurité, et puis c'est vrai que c'est impressionnant tout ce qu'ils ont pu faire il y a des millions d'années, c'est touchant » (FR_2) ;

3/ les détails apportés : "Quand on a vu cette trace de pas humaine ça je trouve impressionnant, moi, cela m'a beaucoup émue, et la petite main avec le petit doigt. Ça montre qu'ils sont passés ici. Et les griffades d'ours aussi ; c'est impressionnant de se dire que les hommes et les ours ont partagé le même lieu de vie » (FR_26) ;

4/ le discours du guide et plus encore ses compétences : «On se laisse guider par le guide, on se laisse emporter. Avec son discours, elle nous a complètement projetés dans le passé. A aucun moment je me suis dit "je ne suis pas dans la vraie". Je suis ressorti impressionné, avec l'impression d'avoir suivi la trace de mes ancêtres » (FR_13) ;

5/ le système de casques audio : «Les caques, ça permet vraiment de rentrer dedans. Ça permet de plus s'isoler, quand on enlevait les casques il y avait les bruits des pas de autres groupes, finalement on voit que ça a aussi participé à mieux se concentrer et à plus regarder ce qu'il y a à voir, à oublier les autres groupes. Le casque, il nous met dans une bulle qui fait qu'on peut se laisser emporter par ce que dit le guide » (FR_11).

Ces artefacts et dispositifs scénographiques donnent corps à la fiction, laquelle, en tant que « jeu partagé (...) suppose simultanément de la part de son destinataire une activité intense de construction de signification à partir des indications trouvées dans le dispositif fictionnel (...). L'attitude de réception attendue de la part du visiteur est donc la suspension des références au monde réel (la suspension d'incrédulité), nécessaire pour qu'il entre dans l'expérience qu'on lui propose de vivre » (Flon, 2012: 72). Cette analyse transversale des entretiens fait ressortir la place centrale occupée par la matérialité dans l'expérience de l'authenticité, rejoignant en cela les recherches de Jones $(2009,2010)$. Ainsi, $96 \%$ des individus interrogés (67 personnes) indiquent leur préférence pour une réplique matérielle par rapport à une réplique virtuelle: «Une réplique physique, c'est plus vivant; cela permet de transmettre plus d'émotions que quelque chose qui serait complètement virtuel » (FR_1) ; «C'est parce qu'on se déplace, parce que notre corps est engagé dans la réplique qu'on a l'impression de visiter la vraie. Faut marcher au milieu de quelque chose pour ressentir une atmosphère » (FR_47).

\section{Limites au ressenti d'authenticité}

Pour les $20 \%$ (14 personnes) n'ayant pas eu le ressenti d'être dans une vraie grotte, auxquels s'ajoutent ceux l'ayant eu ponctuellement (11 personnes), ces limites sont liées à deux facteurs inhérents aux caractéristiques de copie et aux modalités de sa visite.

1/ un défaut d'atmosphère : «Il faudrait retravailler l'atmosphère car là, y'a pas le froid comme quand tu vas dans une vraie grotte. Les volumes, les concrétions, le décor, y'a rien à redire, il est parfait mais il faudrait retravailler l'ambiance »(FR_43). Ce défaut d'atmosphère est 
avancé, indépendamment du fait que les gens aient déjà visité une grotte à concrétions, une grotte ornée ou vu des images de Chauvet en amont de la visite : «Non franchement non je n'ai pas eu l'impression d'être dans une grotte. Non parce que moi j'ai visité des grottes, on n'est pas habillé comme ça déjà. Il fait froid! Au gouffre de Padirac il faut y aller avec un pull et pas avec des tongs sinon ce n'est pas bon, tu te cailles pendant toute la visite » (FR_51). Mais à l'inverse, certains visiteurs apprécient ce confort de visite, comme le montrent les propos de FR_5 : «Il faisait un peu frais à l'entrée, j'ai eu un peu peur que ce soit le cas pendant toute la visite, mais je pense que c'est pour donner l'impression de rentrer dans une vraie grotte après heureusement qu'il ne faisait pas aussi froid qu'à l'entrée. Non il faisait bon, c'est agréable. On a tous les avantages d'une vraie grotte, sans les inconvénients »... avis qui n'est pas sans rappeler ce que P. Bahn (2007 : 141-142) écrivait à propos d'Altamira «le fac-similé, à bien des égards, surpasse l'original ».

Ces résultats contrastés soulignent la dimension relative et individuelle de l'expérience : les perceptions des visiteurs dépendent des parcours de vie de chacun, des expériences individuelles passées, du temps écoulé entre ces expériences et la visite actuelle, de leurs sensibilités respectives, des savoirs intégrés sur l'art pariétal préhistorique et plus largement de leur capacité à avoir incorporé un certain nombre de catégories de perception et de référentiel de ce que doit être la visite d'une grotte du paléolithique, tout en sachant que la majorité d'entre elles sont aujourd'hui fermées à la visite.

2/ le rythme des visites : de manière assez surprenante, le fait d'être en présence d'autres groupes ne ressort pas des entretiens comme un facteur limitatif à la qualité de l'expérience réalisée. Ceci est en partie lié au savoir-faire des guides qui arrivent à réguler la vitesse de déambulation des groupes « de manière à ce que personne ne remarque vraiment que ça pousse derrière ; mais il suffit d'un souci (mauvaise coordination des guides, "bébé hurleur", etc.) pour que ça bouchonne très facilement (surtout en été). Là, tout le monde se sent rapidement à l'étroit » (commentaire d'un guide travaillant à la réplique, été 2017). Ceci est également lié aux éclairages qui masquent pour partie les autres groupes et au port de casque audio qui limite les nuisances engendrées par les autres visiteurs ${ }^{14}$.

La présence des autres groupes est surtout invoquée en raison du rythme de rotation (changement de station toutes les 5 minutes), présenté comme un facteur limitatif à l'expérience d'authenticité. Ainsi, les propos de FR_3: «Quand tu fixes les reproductions, moi, j'ai vraiment oublié où j'étais. Mais en même temps, on ne passe pas assez de temps devant les panneaux. Le panneau du fond, c'est hyper frustrant, on n'a pas le temps de voir les détails que l'autre groupe arrive déjà et nous chasse dehors. S'ils augmentaient le temps passé devant chaque panneau, cela permettrait de plus s'imprégner de toute cette atmosphère »; ou encore EN_1 : "I do not say that the visits should make one hour more, but over the end, have three minutes more for the last stations, it would allow to become soaked more with works. There, it remains superficial, we do not have time to feel completely things. These paintings are 36000 years old and we pass in front of in 5 minutes; I find it limits disrespectful for what our ancestors made ».

Ces citations de visiteurs renvoient aux tensions induites par les différents objectifs poursuivis par la copie : s'il s'agit de donner à faire l'expérience de peintures paléolithiques, il s'agit également de satisfaire des enjeux économiques. L'enchantement se heurte ici aux objectifs de rentabilité visés par le délégataire retenu dans le cadre de la délégation de service public, l'entreprise Kleber-Rossillon. Selon le rythme imposé, l'augmentation des cadences de rotation peut mettre à mal le processus de dénégation dans lequel sont engagés les visiteurs: «l'enchantement est chose fragile, qu'il faut porter à deux [les visiteurs et les concepteurs] pour ne pas risquer la chute » (Lallement \& Winkin, 2015 : 119). A charge pour les concepteurs de 
gérer le plus finement et habilement possible cet équilibre entre le temps nécessaire pour immerger les visiteurs dans l'univers fictionnel proposé et le temps des rotations dont la cadence influe directement sur la rentabilité du site.

\section{Adhésion au dispositif fictionnel et expérience patrimoniale}

En amont de la visite, la volonté des individus à prendre la copie pour la vraie, joue également dans la qualité de l'expérience effectuée. A ce titre, les motivations à venir visiter la réplique témoignent tout autant qu'elles participent à une phase préparatoire à l'enchantement (Winkin, 2001), à des formes d'aveuglements consensuels, où le je sais bien s'efface devant le mais quand même (Lambert, 2013).

Les motivations pour venir visiter la réplique combinent de manière différenciée sept registres. 1/ effet d'opportunité : «On est en vacances dans la région, juste à côté, cela fait partie d'une bonne activité à la journée » (FR_6) ou encore «Parce qu'ils en ont tellement parlé à la télé ! Et puis, c'était sur la route des vacances » (FR_9) ;

2/ intérêt pour l'histoire et de manière générale pour la culture allant de la curiosité : «Tout simplement parce que c'est intéressant de faire autre chose, de se cultiver un minimum et de voir ce qui est intéressant dans la région en dehors des balades et des baignades » (FR_12) à un intérêt très marqué : "That is a part of origins I am fascinated by history and by prehistory thus yes that is a part of subjects which interest me a lot, the history of the humanity in a general way $\gg\left(\mathrm{EN} \_3\right)$;

3/ un devoir de mémoire : «Ça fait partie de notre histoire, c'est notre mémoire, c'est à voir. Faut le voir, c'est tout. C'est épatant et on se rend compte qu'il y a 36000 ans ils avaient tout ce qu'il fallait dans le cerveau » (FR_56) ;

4/ la dimension éducative : «Les enfants voient cela a l'école, alors on est venu pour voir cela en vrai » (FR_41);

5/ être dans une ambiance de grotte : «La curiosité aussi, on avait envie de voir ce que c'était vraiment de voir des peintures rupestres en vrai, dans une vraie grotte » (FR_32) ;

6/ pour les prouesses techniques déployées dans la copie : «Pour voir la performance, voir ce qu'ils ont réussi à faire, il parait que c'était très bien conservé, voir ce qu'on peut reproduire à l'identique c'est vrai que c'est une performance et c'est intéressant de voir ça » (FR_29) ;

7/ entrer en contact avec l'histoire : "Quand une image vous plonge dans le passé c'est assez extraordinaire. La pierre fait le même effet en fait. Visiter un monument du $12^{\mathrm{e}}$ siècle, se dire qu'il y a 1000 ans ils ont fait ça c'est extraordinaire. Ça apporte beaucoup d'humilité de voir ce que notre ancêtre est capable de faire. Avec cette réplique de grotte ornée, c'est pareil, la même démarche, venir voir et ressentir ce que nos ancêtres ont fait »(FR_55).

Si certains des visiteurs cherchent à comprendre l'envers du décor (motivation 6), d'autres recherchent d'entrée de jeu un ressenti d' authenticité et une expérience patrimoniale permettant d'entrer en contact avec l'histoire et de satisfaire des enjeux de transmission (motivations 2, 3, 4, 5 et 7). Ainsi, les propos de FR_60: «Je suis rentrée [dans la réplique] avec cette idée-là, que c'était une vraie ; on peut dire que je me suis bien conditionnée ! Je suis vraiment admirative parce qu'on dirait que ces dessins ils viennent d'être faits alors qu'ils datent de plusieurs milliers d'années. Et puis ce que j'ai aimé c'est que tous ces gens ont fait ces dessins sans avoir l'original sous les yeux. C'est juste de l'observation, ça je suis admirative de ce qu'ils étaient capables de faire, en 36000 ans, on n'a vraiment rien inventé ». Ici, on retrouve les logiques notamment décrites par Ph. Marion : «pour que l'interaction narrative s'épanouisse, il faut que le consommateur de récit entre dans un jeu herméneutique : où veut-on me mener, que veut-on me faire imaginer ou reconstruire du monde, quelle positon dois-je prendre pour entrer dans cette partie narrative ? » (Marion, $1997: 65)$. 
Cette volonté de jouer le jeu et de suspendre volontairement la réalité ne signifie pas pour autant que les visiteurs soient dupes : «Pour entrer en fiction, il faut accepter d'être dupé tout en sachant qu'on l'est. Pour mieux être dedans, il faut qu' une parcelle reste dehors » rappelle $\mathrm{Ph}$. Marion (1997 : 65). A ce titre, le degré de duplicité semble jouer à la marge et savoir que l'on visite une fausse grotte n'empêche pas un ressenti de visite authentique : «Je savais que j'allais visiter une fausse. Et à partir du moment où on sait qu'on va visiter une réplique, c'est quand même difficile de croire qu'on est dans une vraie. Mais cela n'empêche pas d'apprécier la visite et de se laisser prendre au jeu (...) La magie opère, il y a pas de problèmes, on s'y croyait vraiment. Je savais que c'était reproduit et qu'on n'était pas dans la grotte, mais la magie opère, c'est fascinant. La précision des dessins, l'atmosphère, on s'y croirait vraiment » (FR_27).

La dernière phase de l'analyse visait à évaluer la part des personnes ayant eu une expérience de ce qui fait patrimoine, indépendamment de leur ressenti de visiter une vraie grotte. Considérant l'ensemble des réponses ouvertes collectées pour chaque individu, une analyse du verbatim employé pour qualifier la visite de la copie a été effectuée. Sur la base des travaux de Jones (2010: 189) qui met en avant que "When people experience a sense of the genuineness, truthfulness, or authenticity of objects it is something akin to aura, or voicefulness, that they articulate. It is the unique experience of an object, and crucially, I suggest, the web of relationships it invokes with past and present people and places, that is important", les champs lexicaux de l'émerveillement, de l'émotion et du ressenti ainsi que le fait de parler de la copie comme de la vraie ont été considérés comme des marqueurs d'une expérience patrimoniale. Sur la base de cet encodage, $93 \%$ des personnes interrogées ont fait l'expérience de ce qui fait patrimoine ( 65 personnes sur $70^{15}$ ).

Ainsi, les extraits suivants ont été encodés comme marqueurs d'une expérience patrimoniale : EN_4 : «Even if that it is not the real, frankly I was fooled, it is as if I have visited the Chauvet Cave ».

FR_46 : «On se prend une sacrée claque dans la gueule. Franchement les dernières scènes c'est un truc de malade. Je ne m'en suis toujours pas remis. J'en avais la chair de poule surtout les deux derniers arrêts, les chevaux et les lions. J'en étais bouche bée, prêt à baver. Honnêtement j'en ai pris vraiment pleins les yeux ».

FR_57 : «Pour moi c'est vraiment incroyable de voir ce qu'ont fait les premiers hommes. C'est comme si on touchait un peu aux origines et le toucher de près, moi ça me touche aussi ; dès les premières minutes j'ai vraiment ressenti, même si je savais que c'était la reconstitution, ce contact avec les origines, ça m'a vraiment touché, il y'a l'énergie du vieux qui peut être ressentie ».

Ces extraits d'entretien montrent que les visiteurs se sont retrouvés en état d'enchantement, au sens où ils ont volontairement suspendu leur incrédulité le temps de la visite (Winkin, 2001). Le dispositif de la réplique, y compris le discours des guides, a installé une "situation d'enchantement professionnellement organisé » (idem) dans un lieu conçu dans cet objectif. Cet enchantement s'installe d'autant plus facilement que les visiteurs y ont consenti par avance, en faisant la démarche de venir visiter une copie pour «voir des peintures rupestres en vrai » (cf. supra, visiteur FR_32). La rencontre du « dispositif de capture » et de la «bonne volonté des spectateurs » (Lallement \& Winkin, 2015) parachève le processus d'enchantement : les visiteurs ne s'attendent pas à une telle qualité de la restitution et de l'expérience patrimoniale, et cette surprise facilite la bascule vers l'enchantement. L'usage des casques audio, par exemple, qui n'étaient pas prévus au départ, renforce la sensation que le guide confie une sorte de secret à chacun des visiteurs.

Il semble que, dans les sociétés contemporaines, nous soyons habitués à l'hybridation des formes culturelles qui mélangent la fiction et le document : " nous entrons dans le règne d'une 
incrédulité à géométrie variable » (Lambert, 2013 : 118). Cette incrédulité porte sur le contenu du message, et les enquêtes montrent bien que les visiteurs sont passés « maîtres en incrédulité » (idem) : certains doutent que la copie en soit réellement une et voudraient bien pouvoir se convaincre qu'ils ont visité la vraie grotte. Elle porte aussi sur le choix des artefacts, sur les techniques, sur le discours dont chaque élément indique bien que ce ne peut pas être vraiment une copie que l'on visite : « le mystère de nos consommations culturelles (...) est donc celui de nos aveuglements nécessaires » (idem) pour que l'enchantement fonctionne.

\section{Conclusions}

L'analyse des modalités de construction et de réception de la réplique atteste d'une rencontre entre d'une part un univers fictionnel créé par les ingénieurs de l'enchantement (Lallement \& Winkin, 2015 : 118), ou encore les Institutions du Faire Croire (Lambert, 2013 : 140) et d'autre part, des visiteurs prêts à se prendre au jeu de la fiction, des «consommateurs de l'enchantement » qui s'appuyant sur les signes de la narration proposée entrent dans cet univers fictionnel par des opérations de dénégation volontaire de la réalité (Lallement \& Winkin, 2015). La construction de l'authenticité passe par la combinaison de plusieurs paramètres : choix de localisation de la réplique, disposition dans un environnement semi-enterré engageant le corps du visiteur à rentrer sous terre ${ }^{16}$, reconstitution avec finesse des éléments du paysage souterrain (Delannoy et al., 2014), travail sur l'atmosphère en jouant sur la température, les jeux de lumière, et l'intimité produites par les casques audio, et enfin, les procédés narratifs utilisés par les guides de manière à installer les visiteurs dans la vraie grotte. Relevant du domaine du simulacre (Leresche, 2020), la copie vise à produire l'illusion de visiter la vraie grotte en amenant les visiteurs à avoir un ressenti d'authenticité.

Pour que la fiction fonctionne, encore faut-il que les visiteurs adhèrent au récit qui leur est proposé. Sur ce point, l'analyse des modalités de réception par les visiteurs confirme la dimension performative de la copie : les entretiens conduits à la sortie de la réplique auprès des visiteurs attestent que ces derniers, par une suspension volontaire de leur incrédulité, se laissent prendre au jeu et entrent dans le monde du « comme si », avec des gradations de ravissement et parfois même de délectation. En conséquence, la réplique agit ici comme un «powerful locus of authenticity» (Jones, 2010 : 183), et produit «an extremely successful auratic effect» (Foster \& Curtis, $2016: 141)$.

Enfin, l'analyse des verbatims employés par les visiteurs démontre la réalisation d'expériences patrimoniales, où les visiteurs font l'expérience de ce qui fait patrimoine (à la différence de « ce qui est patrimoine », Flon, 2012). Par un effet retour, ces expériences patrimoniales réalisées au sein de la réplique viennent alimenter le processus de patrimonialisation de la grotte originale. La grotte étant fermée pour des raisons de conservation, un risque de décrochage entre les logiques institutionnelles et sociales existe : la possibilité pour les visiteurs de faire une authentique expérience des peintures paléolithiques vient pallier le risque de mise en abîme de la grotte originale, et vient également donner du sens aux deniers publics investis pour conserver un lieu inaccessible (Duval et al., 2020). Dans les années à venir, il s'agira de voir dans quelle mesure les expériences patrimoniales réalisées au sein de la copie conduiront à la définition d'un statut patrimonial de la part des institutions, que viendrait par exemple valider la mise en place de mesures de protection de la copie, de manière à garantir la transmission de cette dernière dans le temps, recoupant ce que Foster and Curtis ont pu relever à propos de copies dans des musées : «as artefacts that are now becoming historic, with their own patinas of age and use - they are things in their own right. They can therefore be of scientific value as examples of craft technologies and practices» (Foster \& Curtis, 2016 : 127). 
Dépassant le contexte des grottes ornées, l'analyse des modalités de construction de la réplique et de sa réception par les visiteurs vient confirmer la place centrale occupée par le ressenti d'authenticité et la dimension expérientielle dans la construction d'un rapport patrimonial. Dans la définition de ce qui fait patrimoine, l'approche essentialiste de l'authenticité est en passe de devenir secondaire par rapport au volet expérientiel, lequel articule des éléments du réel (des vrais guides) et de la fiction (dans une copie de grotte ornée) dans une «feintise ludique partagée » (Schaeffer, 1999). Dans une ontologie sociale du patrimoine (Leveau, 2018), on assiste à une dissociation du triptyque "authenticité, ancienneté et patrimonialisation », laquelle nous invite à aborder la patrimonialisation sous l'angle de l'expérientiel et du ressenti (Tornatore, 2019). Sur ce dernier point, des approches comparatives avec d'autres répliques de grottes ornées (Lascaux II, IV, Altamira, Ekainberri pour ne citer que les européennes), mais également la réalisation d'entretiens avec des visiteurs de grottes ornées ouvertes à la visite (Pech-Merle, Font-de-Gaume, Rouffignac), permettraient de poursuivre la discussion ouverte par l'étude de cas Chauvet, et d'approfondir la place du ressenti d'authenticité dans la construction d'un rapport patrimonial, indépendamment du statut réel ou fictionnel du lieu visité.

Nous remercions vivement Jules Kemper et Françoise Prud'homme pour la qualité de leurs retours, et Xavier Meyer pour son apport dans le traitement de données.

\section{Bibliographie}

Bahn (Paul). 2007. L'art des cavernes. Guide des grottes ornées de la période glaciaire en Europe. Paris : Infolio.

Barthes (Roland). 1968. «L'effet de réel ». Communications, 11, p.84-89.

Baudrillard (Jacques). 1981. Simulacres et simulation. Paris : Galilée.

Cachat (Sylvain), Duval (Mélanie), Gauchon (Christophe). 2012. « Ici, là ou ailleurs ? Les enjeux liés à la localisation d'un grand équipement, l'Espace de restitution de la Grotte Chauvet ». Mondes du Tourisme, 5, p. 13-30.

Davallon (Jean). 2006. Le don du patrimoine, une approche communicationnelle de la patrimonialisation. Paris : Lavoisier.

Delannoy (Jean-Jacques), Sadier (Benjamin), Valcke (Benjamin), Peral (José), Perazio (G.), Jaillet (Stéphane), Tosello (Gilles), Geneste (Jean-Michel) et Clottes (Jean). 2014. «La modélisation 3- D, outil de transfert des connaissances. Exemple de la réplique de la grotte Chauvet : La Caverne du Pont d'Arc (Ardèche France) ». Karstologia, 64, p. 41- 57.

Duval (Mélanie). 2011. «Dynamiques territoriales et développement culturel : la grotte Chauvet comme facteur de réorganisation des gorges de l'Ardèche et outil de légitimation des jeux d'acteurs ?», p. 123-149 in Développement culturel et territoires / sous la direction de Laurent Sébastien Fournier, Catherine Bernié-Boissard, Dominique Crozat et Claude Chastagner. Paris : l'Harmattan.

Duval (Mélanie). 2007. Dynamiques spatiales et enjeux territoriaux des processus de patrimonialisation et de développement touristique. Etude comparée des gorges de l'Ardèche et du Karst slovène. Thèse de Géographie, Laboratoire EDYTEM UMR 5204, Université de Savoie.

Duval (Mélanie), Gauchon (Christophe). 2013. «Le patrimoine : une affaire de choix. La candidature de la grotte Chauvet au patrimoine mondial ». Ardèche Archéologie (30), p. 89-94. Duval (Mélanie), Gauchon (Christophe). 2020, accepté. «The Janus-faced dilemma of rock art heritage management in Europe: a double dialectic process between conservation and public outreach, transmission and exclusion». Conservation and Management of Archaeological Sites 
Duval (Mélanie), Gauchon (Christophe), Smith (Benjamin). 2019. «Rock art Tourism», p.10211041 in The Oxford Handbook of the Archaeology and Anthropology of Rock Art / sous la direction de Bruno David et Ian J. McNivens. Oxford University Press, Oxford.

Eco (Umberco). 1985. La Guerre du faux. Paris: Grasset.

Foster (Sally M.), Curtis (Neil G.W.). 2016. «The Thing about Replicas-Why Historic Replicas Matter». European Journal of Archaeology, 19(1), p. 122-148.

Flon (Émilie). 2012. Les mises en scène du patrimoine, savoir, fiction et médiation. Paris :

Lavoisier.

Gauchon (Christophe). 2009. «Les gorges de l'Ardèche et la grotte Chauvet : redéfinition d'une région touristique ». Téoros, 28 (1), p. 80-92.

Gauchon (Christophe). 2010. Tourisme et patrimoines : un creuset pour les territoires ?. Habilitation à Diriger des Recherches, Laboratoire EDYTEM UMR 5204, Université de Savoie.

Geneste (Jean-Michel), Delannoy (Jean-Jacques), Tosello (Gilles). 2017. « De la grotte à la caverne et de l'archéologie au public ; l'espace de restitution de la grotte Chauvet-Pont d'Arc en Ardèche ». Les Nouvelles de l'Archéologie, 147, p.39-44.

Holtorf (Cornelius). 2013. «On Pastness: A Reconsideration of Materiality in Archaeological Object Authenticity». Anthropological Quarterly, 86 (2), p. 427-443.

James (Nicholas). 2016. « Replication for Chauvet Cave ». Antiquity, 90 (350), p. 519-524.

Jones (Siân). 2009. «Experiencing Authenticity at Heritage Sites: Some Implications for Heritage Management and Conservation». Conservation and mgmt of arch. sites, 11(2), p. $133-$ 147.

Jones (Siân). 2010. «Negotiating Authentic Objects and Authentic Selves: Beyond the Deconstruction of Authenticity». Journal of Material Culture, 15 (2), p. 181-203.

Jones (Siân), Yarrow (Thomas). 2013. «Crafting Authenticity: An Ethnography of Conservation Practice». Journal of Material Culture, 18 (1), p. 3-26.

Kaeser (Marc-Antoine). 2011. L'âge du faux. Neuchâtel : Laténium.

Kaminski (Jaime). 2014. «Decorated Palaeolithic cave sites as a tourism resource: the Franco cantabrian perspective», p. 165-176 in Contemporary Issues in Cultural Heritage Tourism / sous la direction de Jamie Kaminski, Angela M Benson et David Arnold. London, New York: Routledge.

Labadi (Sophia). 2010. «World Heritage, authenticity and post-authenticity: International and national perspectives », p. 66-84 in Heritage and Globalisation. Key Issues in Cultural Heritage / sous la direction de Sophia Labadi et Colin Long. London, New York: Routledge.

Lallement (Emmanuelle) et Winkin (Yves). 2015. «Quand l'anthropologie des mondes contemporains remonte le moral de l'anthropologie de la communications ». Communiquer, 13, p. $107-122$.

Lambert (Frédéric). 2013. Je sais bien mais quand même: essai pour une sémiotique des images et de la croyance. Le Havre : Éd. non standard

Latour (Bruno), Lowe (Adam). 2011. «The Migration of the Aura, or How to Explore the Original through its Facsimiles», p. 275-298 in Switching Codes: Thinking Through Digital Technology in the Humanities and the Arts / sous la direction de Thomas Bartscherer et de Roderick Coover. Chicago, IL: University of Chicago Press.

Leresche (Nicolas). 2020. "From the Lascaux cave to Lascaux IV, repetition and transformation of a simulacrum », p. 137-1555 in Tourism fictions, simulacra and virtualities / sous la direction de Maria Gravari-Barbas, Nelson Graburn et Jean-François Staszak. Oxon, New York: Routledge.

Leveau (Pierre). 2018. «L'ontologie sociale du patrimoine : Lascaux et le problème du temps 》. Nouvelle revue d'esthétique, 21, p. 51-60. 
Levent (Nina), A. Pascual-Leone (Alvaro). 2014. The multisensory museum: cross-disciplinary perspectives on touch, sound, smell, memory and space. Plymouth: Rowman \& Littlefield. Malgat (Charlotte). 2016. Tourisme de qualité, diversification touristique et trajectoires territoriales du sud Ardèche. Mise en place d'indicateurs IQUATEXT appliqués au grand projet grotte Chauvet-Pont-d'Arc. Thèse de Géographie, Laboratoire EDYTEM UMR 5204, Université de Savoie.

Malgat (Charlotte), Duval (Mélanie). 2014. « La labellisation Unesco de la grotte Chauvet, une démarche internationale pour une reconfiguration locale », p. 395-409 in Labellisation et mise en marque des territoires / sous la direction de Mauricette Fournier. Clermont-Ferrand : Presses Universitaires de Blaise Pascal.

Malgat (Charlotte), Duval (Mélanie), Gauchon (Christophe). 2012. «Donner à voir un patrimoine invisible : de l'original à la copie. Le cas de l'Espace de restitution de la Grotte Chauvet », p. 99-114 in Karsts - Paysages et Préhistoire / sous la direction de Jean-Jacques Delannoy, Stéphane Jaillet et Benjamin Sadier. Chambéry : Collection Edytem, 13.

Malgat (Charlotte), Duval (Mélanie), Gauchon (Christophe). 2015. « Fac-similés et transfert de patrimonialité. La grotte ornée Chauvet-Pont-d'Arc ». Culture \& Musées, 25, p. 141-163.

Marion (Philippe). 1997. «Narratologie médiatique et médiagène des récits ». Recherches en communication, 7, p. 61-88.

Pieraccini (Massimiliano), Guidi (Gabriele), Atzeni (Carlo). 2001. «3D digitizing of cultural heritage ». Journal of Cultural Heritage, 2, p. 63-70.

Pinçon (Geneviève), Geneste (Jean-Michel). 2010. «Art rupestre : la 3D un outil de médiation du réel invisible? » In Situ, 13, s.p.

Schaeffer (Jean-Marie). 1999. Pourquoi la fiction ? Paris : Le Seuil.

Smith (Laurajane). 2006. Uses of Heritage. London: Routledge and Taylor \& Francis Group.

Stone (Peter G.), Planel (Philippe G.). 1999. «Introduction Re-construction VS construction », p.1-14 in The constructed Past, experimental archaeology, education and the public/ sous la direction de Peter G. Stone et Philippe G. Planel. London, New York: Routledge.

Stylianidis (Efstratios), Remondino (Fabio). 2016. 3D recording, documentation and management of cultural heritage. Dunbeath: Whittles Publishing.

Tornatore (Jean-Louis). 2019. "Introduction Expériencer le patrimoine », p.9-65 in Le patrimoine comme expérience, Implications anthropologiques/sous la direction de Jean-Louis Tornatore. Paris : éditions de la Maison des sciences de l'homme.

Valladas (Hélène), Clottes (Jean), Geneste (Jean-Michel), Garcia (M.), Arnold (M.), Cachier H. et Tisnérat - Laborde (Nadine). 2001. «Evolution of prehistoric cave art ». Nature, 413, p. 479.

Winkin (Yves). 1998. «Le touriste et son double. Éléments pour une anthropologie de l'enchantement », p. 133-143 in Miroirs maghrébins: itinéraires de soi et paysage de rencontre/ sous la direction de Susan Ossman. Paris : éd. CNRS.

Winkin (Yves). 2001. «Propositions pour une anthropologie de l'enchantement», p. 169-179 in Unité-diversité : les identités culturelles dans le jeu de la mondialisation/ sous la direction de Paul Rasse, Nancy Midol et Fathi Triki. Paris : L'Harmattan.

\section{Sources}

SMERGC (Syndicat Mixte de l'Espace de Restitution de la Grotte Chauvet-Pont-d'Arc). 2012. La grotte Chauvet Pont d'Arc, Dossier de presse "Pose de la Première main - Espace de restitution $», 22 \mathrm{p}$.

\section{Notices biographiques}


Mélanie Duval, chargée de recherche CNRS, laboratoire EDYTEM UMR 5204 CNRS /

Université Savoie Mont Blanc ; chercheure associée au Rock Art Research Institute, School

of Geography, Archaeology and Environmental Studies, University of the Witwatersrand

Ses recherches portent sur les enjeux liés au processus de patrimonialisation de lieux ayant une dimension archéologique (sites d'art rupestre, vestiges lacustres). Elle s'intéresse aux valeurs mobilisées et aux jeux d'acteurs dans la construction et le fonctionnement de la rhétorique patrimoniale, ainsi qu' aux modes de mise en valeur et pratiques touristiques. En co-auteur avec Christophe Gauchon et Benjamin Smith, elle a publié en 2019 un chapitre intitulé « Rock art Tourism », dans l'ouvrage de référence Anthropology of Rock Art, dirigé par Bruno David et d'Ian McNiven.

melanie.duval@univ-smb.fr

ORCID : https://orcid.org/0000-0003-3570-3104

Christophe Gauchon, professeur des Universités, Université Savoie Mont Blanc / laboratoire EDYTEM UMR 5204 CNRS.

Il a étudié la fréquentation et l'aménagement des cavernes dans les montagnes françaises puis a étendu son champ d'analyse vers les développements du tourisme, les processus de patrimonialisation et les logiques de protection, avec un intérêt particulier pour la place et l'usage de la toponymie dans les logiques patrimoniales. En co-auteur avec Mélanie Duval et Benjamin Smith, il a publié en 2019 un chapitre intitulé « Rock art Tourism », dans l'ouvrage de référence Anthropology of Rock Art, dirigé par Bruno David et d'Ian McNiven.

christophe.gauchon@univ-smb.fr

Charlotte Malgat, docteure en géographie, chercheure associée au laboratoire EDYTEM UMR 5204 CNRS / Université Savoie Mont Blanc.

Soutenue en 2016, sa recherche doctorale visait à comprendre et à analyser la réorganisation touristique du territoire sud-ardéchois suite à la mise en place des projets de valorisation de la grotte Chauvet-Pont-d'Arc (réalisation du fac-similé / inscription au Patrimoine mondial). Dans le cadre de missions post-doctorales, elle a continué à analyser les logiques de marquage territorial dans le sud-Ardèche via l'utilisation par les acteurs territoriaux des labels tout en participant à l'analyse des modalités de réception de la copie de la grotte Chauvet-Pont d'Arc par les publics. En co-auteur avec Mélanie Duval, elle a notamment publié en 2014 «La labellisation Unesco de la grotte Chauvet, une démarche internationale pour une reconfiguration locale », dans l'ouvrage Labellisation et mise en marque des territoires, dirigé par Mauricette Fournier.

charlotte.malgat@hotmail.fr

Une seule et même adresse postale :

Laboratoire Edytem UMR 5204

Université de Savoie

Pôle Montagne

Campus scientifique de Technolac

73376 Le Bourget du Lac Cedex

1 https://france3-regions.francetvinfo.fr/auvergne-rhone-alpes/ardeche/caverne-du-pont-arc-prend-nom-grottechauvet-2-ardeche-1618707.html 
2 Le site se compose d'un espace d'accueil, une boutique, un restaurant, un espace évènementiel où se déroulent des spectacles et autres animations sur le thème de la préhistoire, un espace pédagogique pour accueillir le jeune public, la copie à proprement parler et un centre d'interprétation baptisé « Galerie de l'Aurignacien ».

3 S'il s'agit pour partie d'une captation de touristes déjà présents sur le territoire, l'Agence de Développement Touristique de l'Ardèche estime que, entre 2015 et 2017, la réplique a déclenché la venue de 280000 touristes supplémentaires, et généré un impact net sur le territoire de 80 millions d'euros.

4 La philosophie rejoint ici des réflexions également développées par d'autres Sciences Humaines et Sociales où la patrimonialisation est abordée en tant qu'entité communicationnelle (Davallon, 2006 ; Flon, 2012) ou encore de construit sociospatial et culturel (Gauchon, 2010 ; Jones 2009 ; Smith 2006).

5 https://www.francetvinfo.fr/sciences/je-pensais-que-la-grotte-chauvet-serait-incopiable-mais-on-1-afait_857603.html, consulté le 25/07/2018

6 «Nous, nous voulions que, lorsque les visiteurs entrent, ils se sentent comme dans une vraie grotte, pas dans un décor. Je voulais reproduire la grotte dans ses moindres détails » (interview de Mélanie Claude, 04/05/2015, France TV Info) ; https://www.francetvinfo.fr/sciences/je-pensais-que-la-grotte-chauvet-serait-incopiable-mais-on-1-a-fait_857603.html, consulté le 25/07/2018

$7 \quad$ Un éclairage diffus éclaire les panneaux le temps de présence du groupe à chaque station ; l'ambiance de pénombre dépend pour beaucoup de nombre de groupes présents en même temps dans la copie (jusqu'à 10 groupes), et du degré de covisibilité entre les stations.

$8 \quad$ Par groupe de 20 à 28 personnes maximum, le visiteur chemine dans la copie le long du parcours prévu à cet effet pendant 50 minutes. A l'entrée, chaque visiteur se voit remettre un casque audio réglé sur la même fréquence que le microémetteur du guide chargé du groupe. Ce dispositif a été mis en place à l'été 2016, un an après l'ouverture de la copie pour éviter la gêne occasionnée par la proximité entre les groupes.

9 Deux d'entre elles ont été effectuées en écoutant des commentaires préenregistrés en langue anglaise au sein de groupes francophones; les 20 visites restantes ont été faites en écoutant les commentaires délivrés en direct par le guide. Pour les touristes étrangers, des visites guidées en anglais, en allemand et en hollandais sont proposées à heure fixe ; à défaut, s'ils font partie d'une visite en français, des audio-guides avec des discours préenregistres en plusieurs langues leur sont proposés. Les deux modes de visite, visite guidée avec des commentaires en direct (mode 1) versus visite avec un audio-guide préenregistré (mode 2) ne donnent pas lieu à la même expérience. Ecouter l'audio-guide préenregistré instaure de facto un décalage entre le discours tenu au même moment par le guide français au reste du groupe et celui dispensé en anglais par l'appareil. Suite aux discussions avec les guides travaillant sur le site, il apparaît que les audio-guides sont mal reçus par les visiteurs : les explications données restent superficielles par rapport à celles délivrées dans le même temps par le guide en présentiel, le discours ne suit pas le même déroulé que celui du guide qui montre des éléments dans les peintures qui ne sont pas abordées dans le même temps sur la bande préenregistrée, et au final, les visiteurs ayant un audio-guide tendent à évoluer en «électrons libres difficiles à gérer » (commentaire d'un guide travaillant dans la restitution, été 2017).

10 Les guides officiant dans la restitution (une cinquantaine à l'année, près de 80 en période estivale) présentent des profils très diversifiés, certains venant de l'histoire (des médiévistes pour l'essentiel), de l'histoire de l'art, du tourisme, de la médiation scientifique ou de secteurs complètement différents. Sur ce total, une dizaine d'entre eux ont un bagage en tant que préhistorien. A leur prise de fonction, une formation de quatre jours leur est dispensée, laquelle prend la forme d'un enseignement de culture générale sur la préhistoire, avec quelques focus sur les Aurignaciens, l'art pariétal et la grotte Chauvet. Durant cette période, ils suivent également des guides déjà en poste afin de s'approprier le discours tenu et les techniques de médiation employées. C'est sur cette base, et en prenant également appui sur leurs parcours de vie et leur formation, qu'ils vont progressivement construire leur propre discours en direction des visiteurs dont ils auront la charge, ce qui explique les différences d'explication de certains panneaux, les uns mettant plus l'accent sur les techniques, les autres sur le registre émotionnel, etc.

11 Des entretiens semi-directifs ont été conduits avec un panel constitué de 70 individus (60 francophones; 10 anglophones). Ce choix de diversifier les individus selon leur provenance s'explique compte tenu de la fréquentation globale du site ( $80 \%$ de visiteurs sont français, suivis par les Belges et les Hollandais, puis les Anglais et les Allemands dans une moindre mesure). Seuls des individus ayant effectué une visite guidée avec le guide (mode 1) ont été interrogés. Aussi, ce travail ne permet pas d'appréhender les modalités de réception de la copie par les visiteurs étrangers ayant eu une visite avec un audio guide préenregistré, lesquelles sont a priori différentes. D’une durée de 10 à 15 minutes, les entretiens ont été réalisés à la sortie de la copie et se composent d'une alternance de questions ouvertes / fermées portant sur : motivations à venir en Ardèche ; nombre de séjours effectués ; activités pratiquées ; intérêt pour visiter le site de la réplique ; précédentes visites de sites ornés ou de copies ; ressenti lors de la visite de la copie ; positionnement par rapport aux répliques virtuelles. L'objectif était d'obtenir un panel de réponses le plus diversifié possible, sans pour autant suivre une logique d'échantillonnage propre aux approches quantitatives. Aussi, si les enquêtrices (MD et $\mathrm{CM}$ ) ont cherché à diversifier les profils selon trois principales modalités (âge, genre, composition des groupes), le corpus d'individus (70) ne permet pas une approche quantitative, laquelle 
aurait au demeurant peu de sens pour questionner des perceptions. Les pourcentages donnés dans la suite de l'étude visent à contextualiser les résultats de l'enquête, et ne peuvent pas être extrapolés pour qualifier de manière globale les publics fréquentant le site de la réplique. Enregistrés, anonymés puis retranscrits, leur analyse permet une analyse de contenu des modalités de réception de la copie.

$1280 \%, 56$ personnes

$1320 \%$ des personnes ayant eu le ressenti d'être dans une grotte, soit 11 personnes

14 Sur ce point, les résultats auraient très certainement été très différents si les enquêtes avaient eu lieu au lendemain de l'ouverture du site en avril 2015 où les commentaires de guides étaient diffusés à haute voix dans la réplique (observations terrain avril 2015 ; James, 2016).

15 Sur les 5 personnes n'ayant pas eu le ressenti de ce qui fait patrimoine, trois d'entre eux sont des entretiens très courts (5 minutes) et le matériel récolté est assez pauvre. Les deux restants ont été très frustrés par le manque d'ambiance grotte (FR_58) et par le rythme de la visite (FR_24), ces deux facteurs ayant fortement limité la qualité de leur expérience.

16 A ce titre, voir également les développements de Nicolas Leresche (2020) sur les copies de la grotte de Lascaux. 